\title{
Dynamic redistribution of the electric field of the channel in AIGaN/GaN high electron mobility transistor with nanometer-scale gate length
}

\author{
S. A. Vitusevich, ${ }^{\text {a) }}$ S. V. Danylyuk, A. M. Kurakin, N. Klein, and H. Lüth \\ Institut für Schichten und Grenzflächen and CNI-Center of Nanoelectronic Systems for Information \\ Technology, Forschungszentrum Jülich, Jülich D-52425, Germany
}

M. V. Petrychuk

Taras Shevchenko National University, Kiev 01033, Ukraine

\author{
A. E. Belyaev \\ V. Lashkaryov Institute of Semiconductor Physics, NASU, Kiev 03028, Ukraine
}

(Received 29 December 2004; accepted 12 September 2005; published online 3 November 2005)

\begin{abstract}
Transport peculiarities and the physical origin of noise properties in $\mathrm{AlGaN} / \mathrm{GaN}$-based high electron mobility transistors (HEMTs) with a large ratio of channel length to gate length were investigated. Dependence of deviations of low-frequency noise spectra from the $1 / f$ law on applied gate voltages was studied in an extended range of frequencies. The behavior is explained in terms of a model based on the dynamic redistribution of the electric field along the two-dimensional channel of the HEMT. The results show that the main contribution to the noise originates from the region under the gate and adjacent to the gate channel regions. () 2005 American Institute of Physics. [DOI: 10.1063/1.2128067]
\end{abstract}

Modern nanotechnology not only allows a considerable decrease in the characteristic size of the investigated material objects and effective control of their properties, but also introduces novel features and effects in the transport and noise properties of the objects. For our particular case, scaling the gate length to nanosize results in an increase of the operating frequency of high electron mobility transistors (HEMTs). At the same time, increasing the ratio of $L_{\mathrm{SD}} / L_{G}$, where $L_{\mathrm{SD}}$ and $L_{G}$ are distances between source and drain and gate length, respectively, enhances the role of regions outside the gate. Therefore, investigations of transport peculiarities and the physical origin of noise properties in HEMTs with a large ratio of $L_{\mathrm{SD}} / L_{G}$ are extremely important. Recently, we have demonstrated how to separate the contribution from the passive and active regions of HEMTs with nanoscale gate length to the total noise level. ${ }^{1}$ In this letter, we report results concerning novel peculiarities observed in output and transfer characteristics and noise spectra of nanoscale gate HEMT structures $\left(L_{\mathrm{SD}} / L_{G}>10\right)$.

The investigated structures were designed for highfrequency and low-noise applications and a high-density two-dimensional electron gas (2DEG) was created without doping-utilizing piezo- and spontaneous polarization effects in GaN/AlGaN heterostructures. Each structure consists of the following layers grown by metalorganic vapor phase epitaxy on a sapphire substrate: a nucleation AlGaN $(16 \% \mathrm{Al})$ layer, an undoped $\mathrm{GaN}$ buffer layer, and an undoped AlGaN (33\% Al) barrier layer. The surface of the devices was passivated with $\mathrm{Si}_{3} \mathrm{~N}_{4}$. Devices with several gate lengths $(150-350 \mathrm{~nm})$ and widths $(100-400 \mu \mathrm{m})$ were investigated. A description of the preparation of the structures, as well as the characterization of the materials and devices studied, has been reported elsewhere. ${ }^{1}$ Output and transfer characteristics and noise spectra were measured in a wide temperature range $(45 \mathrm{~K}-300 \mathrm{~K})$.

\footnotetext{
${ }^{a)}$ Electronic mail: s.vitusevich@fz-juelich.de
}

Peculiarities of low-frequency noise in $\mathrm{AlGaN} / \mathrm{GaN}$-based HEMTs were reported in our previous paper. $^{2}$ The major feature is the appearance of the region with a $1 / f^{\gamma}$ slope, where $\gamma=3 / 2$ under increasing gate bias, in the samples with low leakage current against $1 / f$ noise background. This feature becomes most pronounced with decreasing gate length. It is clearly seen in Fig. 1, where normalized current noise spectra measured at a variety of gate biases are presented.

The noise spectra were measured in the frequency range of $1 \mathrm{~Hz}-100 \mathrm{kHz}$ in the transistors' linear operating regime. At drain-source voltages of up to $100 \mathrm{mV}$, the currentvoltage characteristics demonstrate a linear dependence. The linearity is also conserved when the temperature is reduced to $45 \mathrm{~K}$. The channel resistance decreases with decreasing temperature mainly due to the growth of mobility because the carrier concentration remains practically unchanged.

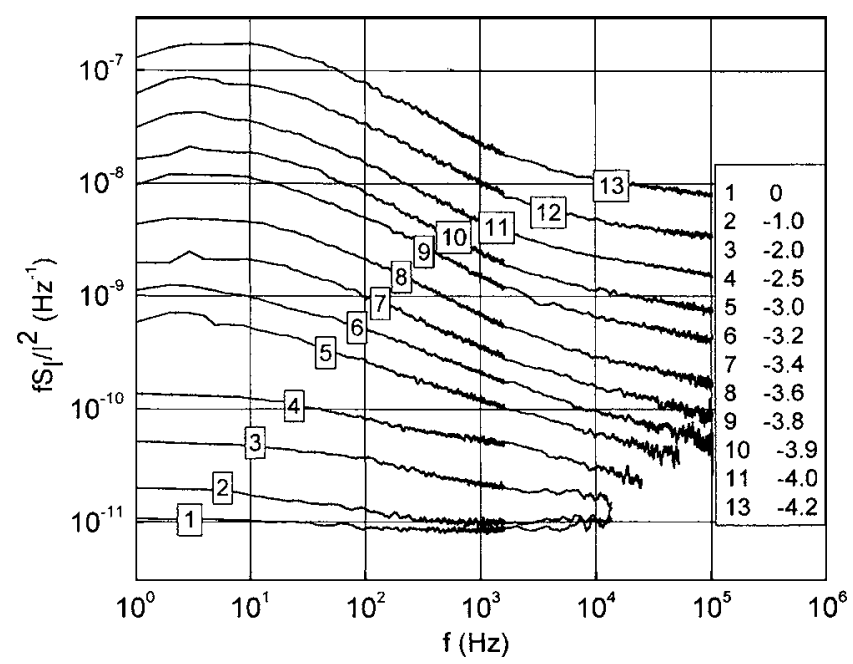

FIG. 1. Normalized current noise spectra measured at room temperature and different gate voltages. Gate length is $250 \mathrm{~nm}$, spacing between source and drain is $3 \mu \mathrm{m}$. $V_{\mathrm{DS}}=100 \mathrm{mV}$. 

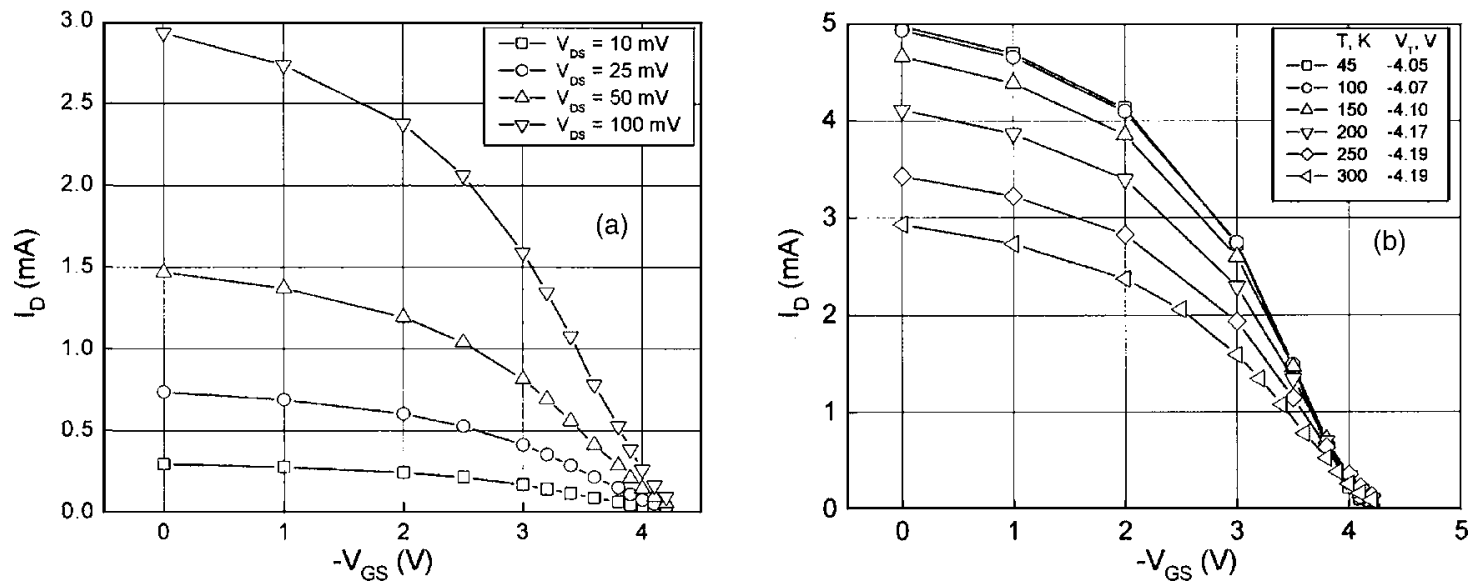

FIG. 2. Transfer characteristics of the transistor measured: (a) at room temperature and different $V_{\mathrm{DS}}$ and (b) at different temperatures and $V_{\mathrm{DS}}=100 \mathrm{mV}$.

Transfer characteristics of the devices studied, shown in Fig. 2 , confirm the latter. It is seen that the variation of the threshold voltage is $3 \%$ in the temperature interval from $45 \mathrm{~K}$ to $300 \mathrm{~K}$. In the linear regime, the total resistance of the channel demonstrates an inversely proportional dependence on gate voltage, $V_{G}$. It is convenient to use normalized effective gate voltage to threshold voltage, $V_{T}: V_{G n}=\left(V_{G}\right.$ $\left.-V_{T}\right) /\left|V_{T}\right|$, and present the total channel resistance as a sum: $R=R_{p}+R_{a}$, where $R_{p}$ comprises the resistance of that part of the channel outside the gate metallization (passive region) and $R_{a}$ is the resistance under the gate (active region). ${ }^{1}$ Measurements on transmission line model structures showed that contacts did not contribute much to the total resistance $(10 \%$ of total resistance), and their influence can be neglected. As seen in Fig. 3, the total resistance follows $1 / V_{G n}$ dependence fairly well. Because the gate voltage affects the active region only, the changes in $R$ are due entirely to the changes in $R_{a}$. Deviations are observed only at very small negative gate voltages, where $R_{a} \ll R_{p}$, and the total resistance approaches the invariable background (total resistance of the channel at $V_{G}=0$ ), and at gate voltages close to pinch-off, where changes in carrier mobility should be expected due to the hot electron effect in the strongly nonuniform high electric field.

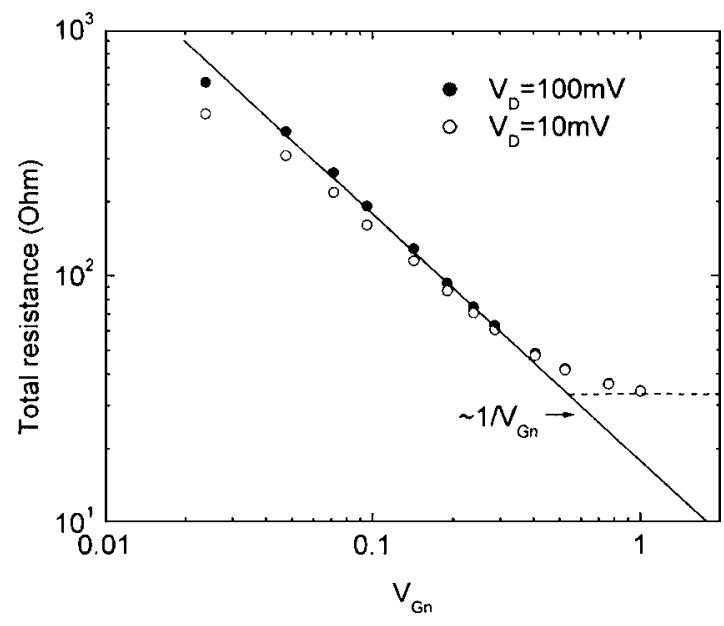

FIG. 3. Total resistance of the channel as a function of normalized effective gate voltage. Symbols are experimental data obtained at $V_{\mathrm{DS}}=10 \mathrm{mV}$ (open circles) and $V_{\mathrm{DS}}=100 \mathrm{mV}$ (solid circles). $T=300 \mathrm{~K}$. The solid line shows $1 / V_{G n}$ dependence, and the dashed line determines invariable background

the active region, we include not only part of the conducting
(resistance of passive region).
Downloaded 21 Dec 2006 to 134.94.122.39. Redistribution subject to AIP license or copyright, see http://apl.aip.org/apl/copyright.jsp
In order to analyze the features observed in the noise spectra, we assume that the noise sources in the passive and active regions of the channel are uncorrelated. In this case, the following expression for the current noise density can be written as ${ }^{3}$

$$
\frac{S_{I}}{I^{2}}=\frac{S_{R}}{R^{2}}=\frac{S_{R a}+S_{R p}}{R^{2}}=\frac{\alpha_{a} e \mu R_{a}^{3}}{L_{a} f\left(R_{a}+R_{p}\right)^{2}}+\frac{\alpha_{p} e \mu R_{p}^{3}}{L_{p} f\left(R_{a}+R_{p}\right)^{2}},
$$

where $\alpha, \mu$, and $L$ are the Hooge parameter, carrier mobility, and channel length, respectively. $e$ is the electronic charge and subscripts $a$ and $p$ correspond to active and passive regions. The ratio of the resistance and power of noise in active and passive regions will determine the effect of the gate voltage on the current noise density. If the gate region dominates, i.e., $R_{a} \gg R_{p}, S_{R a} \gg S_{R p}$ and taking into account the dependence $R_{a} \sim 1 / V_{G}$, Eq. (1) easily transforms to

$$
\frac{S_{I}}{I^{2}}=\frac{S_{R a}}{R_{a}^{2}} \sim \frac{1}{V_{G}}
$$

In the opposite case, when the passive region dominates, i.e., $R_{a} \ll R_{p}, S_{R a} \ll S_{R p}$, the current noise density is expected to be constant. Two other cases concern intermediatem conditions. If the noise source of the active region remains more powerful $\left(\mathrm{S}_{R a} \gg \mathrm{S}_{R p}\right)$, while its resistance is much smaller than $R_{p}$, Eq. (1) yields the following approximation:

$$
\frac{S_{I}}{I^{2}}=\frac{S_{R a}}{R_{p}^{2}} \sim \frac{1}{V_{G}^{3}} .
$$

And finally, at large negative gate voltages close to pinch off, where $R_{a} \gg R_{p}$ and assuming that resistance fluctuations are determined by the passive region $\left(S_{R a} \ll S_{R p}\right)$, we obtain

$$
\frac{S_{I}}{I^{2}}=\frac{S_{R p}}{R_{a}^{2}} \sim V_{G}^{2} .
$$

A comparison of the experimental data plotted in Fig. 4, with the approximations presented above, shows that in the entire range of the gate voltages the cases described by Eqs. (2) and (3) are the most realistic. The latter allows us to conclude that in spite of the large ratio of $L_{\mathrm{SD}} / L_{G}$, the active region of the device is the main contributor to the observed lowfrequency noise. It should be noted that when speaking about the active region, we include not only part of the conducting 


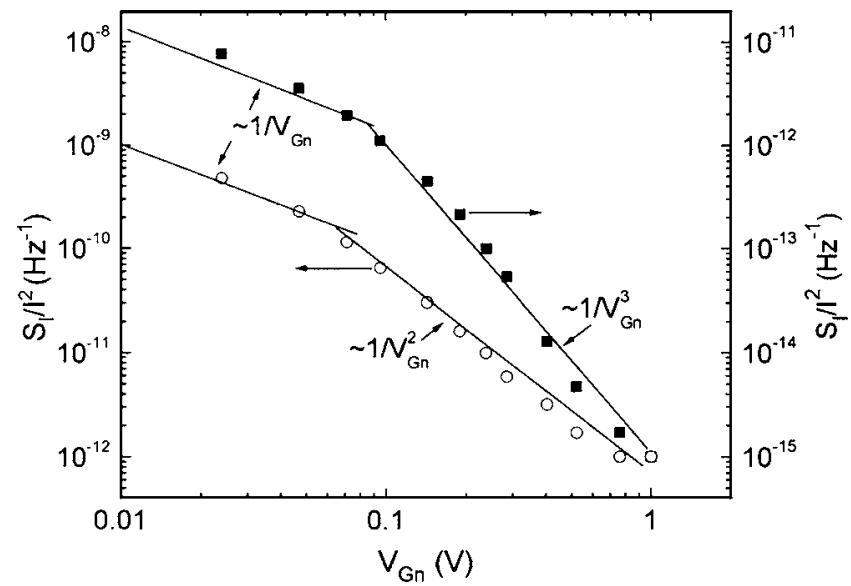

FIG. 4. Dependences of current noise density on normalized effective gate voltage. Symbols are experimental data obtained at $10 \mathrm{~Hz}$ (open circles) and $10 \mathrm{kHz}$ (solid squares). $T=300 \mathrm{~K}$. The solid lines show for convenience different power dependences on gate voltage.

channel under the gate, but also adjacent parts of the sourceto-gate and gate-to-drain regions. It is seen from Fig. 4 that it is the threshold gate bias, $V_{G t} \approx-3 \mathrm{~V}$, which distinguishes different regimes of the transistor operation with weak and strong control of the channel resistance.

Finally, we would like to discuss the observed peculiarities in $1 / f$ noise spectra. Conventional generationrecombination processes cannot explain the behavior observed. ${ }^{4}$ In our opinion, a model based on the dynamic redistribution of lateral potential along the conducting channel is more relevant. A static approach to such redistribution has been developed in Ref. 5. The authors of Ref. 6 also propose a model based on the existence of lumped-thermalnoise generators along the semiconductor-air or semiconductor-dielectric protection interface. In this letter, we consider the existence of an additional conductive channel located either on the surface or in the barrier layer at a short distance from the basic 2DEG channel. Between the 2DEG and additional channels, strong electrostatic coupling should appear. At high enough electric fields (at a large gate voltage, the electric field in the active region approaches several $\mathrm{kV} / \mathrm{cm}$ ), the difference in lateral distribution of the potential in the basic and additional channels leads to charge redistribution in the additional channel. The latter should determine the redistribution of the transverse potential that supports $2 \mathrm{DEG}$ in the basic channel. As a result, lateral distribution of electron density in the basic channel is changed. In our model, intrinsic density fluctuations in the 2DEG play the role of dynamic inhomogeneities. These fluctuations are associated with electrons in the additional channel which pull them along. At low frequencies, this effect causes "slow" electrons of the additional channel to contribute to $1 / f$ noise together with fast electrons of the basic channel, which causes an increase in the $1 / f$ noise level. At high frequencies, slow electrons of the additional channel cannot respond to fast fluctuations of the 2DEG. In this case, there is no extra contribution to the $1 / f$ noise. Thus, from analyses of the noise spectra, we can conclude that the low-frequency part of the $1 / f$ noise contains high-frequency fast electron fluctuations, which are caused by the dynamic potential redistribution at certain gate voltages in basic and additional channels.

In conclusion, two characteristic gate voltage ranges were established, separated by different ratios of resistances and noise sources of the passive and active regions along the channel of the HEMT. An important contribution of the active region to the low-frequency part of noise spectra with increasing gate voltage was observed in the linear regime. Observed peculiarities in the $1 / f$ noise spectra were explained within the framework of a model based on the dynamic redistribution of lateral potential along the conducting channel.

This work is supported by Deutsche Forschungsgemeinschaft (Project KL 1342).

${ }^{1}$ S. A. Vitusevich, S. V. Danylyuk, N. Klein, M. V. Petrychuk, V. N. Sokolov, V. A. Kochelap, A. E. Belyaev, V. Tilak, J. Smart, A. Vertiatchikh, and L. F. Eastman, Appl. Phys. Lett. 80, 2126 (2002).

${ }^{2}$ S. A. Vitusevich, S. V. Danylyuk, N. Klein, M. V. Petrychuk, V. N. Sokolov, V. A. Kochelap, A. E. Belyaev, V. Tilak, J. Smart, A. Vertiatchikh, and L. F. Eastman, Proc. ICPS26 (2002).

${ }^{3}$ J.-M. Peransin, P. Vignaud, D. Rigaud, and L. K. J. Vandamme, IEEE Trans. Electron Devices 37, 2250 (1990).

${ }^{4}$ F. N. Hooge, Physica B 311, 238 (2002).

${ }^{5}$ B. K. Ridley, J. Appl. Phys. 90, 6135 (2001).

${ }^{6} \mathrm{M}$. Poudysegur, J. Graffeuil, and J.-L. Cazaux, IEEE Trans. Electron Devices 34, 2178 (1987). 